\title{
Differences in Helicobacter pylori CagA tyrosine phosphorylation motif patterns between western and East Asian strains, and influences on interleukin-8 secretion
}

Correspondence

Richard H. Argent

richard.argent@nottingham.ac.uk

Received 10 March 2008

Accepted 30 May 2008

\author{
Richard H. Argent, ${ }^{1,2,3}$ † James L. Hale, ${ }^{1,2} \dagger$ Emad M. El-Omar ${ }^{4}$ \\ and John C. Atherton ${ }^{1,2}$
}

${ }^{1}$ Institute of Infection, Immunity and Inflammation, Centre for Biomolecular Sciences, University of Nottingham, Nottingham NG7 2RD, UK
${ }^{2}$ Wolfson Digestive Diseases Centre, Queen's Medical Centre, University of Nottingham, Nottingham NG7 2UH, UK
${ }^{3}$ Division of Pre-Clinical Oncology, Queen's Medical Centre, University of Nottingham, Nottingham NG7 2UH, UK
${ }^{4}$ Department of Medicine and Therapeutics, Institute of Medical Sciences, University of Aberdeen, Foresterhill, Aberdeen AB25 2ZD, UK

\begin{abstract}
Helicobacter pylori strains from East Asia have an 'East Asian' type of CagA that is more active and predominantly comprises a single type. Strains from other countries have a 'western' type of CagA, which is less active and comprises many different types generated by intragenomic recombination. Co-culture of AGS gastric epithelial cells with isolates of western strains that displayed microevolution in CagA showed that isolates with additional copies of the $\mathrm{C}$ motif induced significantly more interleukin (IL)-8 secretion. Co-culture of AGS cells with western and East Asian strains, each expressing CagA with a single copy of the C or D motif, showed that East Asian strains induced significantly more IL-8 secretion. Analysis of the different CagA types from data deposited in GenBank and from the literature showed that western CagA is significantly more likely to undergo duplication of tyrosine phosphorylation motif $\mathrm{C}$ than East Asian CagA is of the corresponding $D$ motif. Taken together, the data suggest that the already highly active East Asian CagA with one $D$ motif has no requirement to increase its virulence, whereas the less active western CagA displays flexibility in its capacity to increase its number of tyrosine phosphorylation motifs to become more virulent.
\end{abstract}

\section{INTRODUCTION}

Helicobacter pylori infects approximately half of the world's population and is the leading cause of gastric and duodenal ulceration and gastric cancer (Atherton, 2006). Around $70 \%$ of strains possess the cagA gene, a marker of the cag pathogenicity island (PAI), although prevalence is more than $90 \%$ in East Asian countries. The presence of cagA has been associated with the development of gastric cancer (Blaser et al., 1995). CagA is transported through the type IV secretion system encoded by the cag PAI into the hostcell cytosol, where it becomes phosphorylated by Src and

†These authors contributed equally to this work.

Abbreviations: cag PAl, cytotoxin-associated gene pathogenicity island; $\mathrm{Cl}$, confidence interval; Csk, C-terminal Src kinase; IL-8, interleukin-8; $\mathrm{OR}$, odds ratio; RAPD-PCR, random amplified polymorphic DNA PCR; TPM, tyrosine phosphorylation motif; VR, $\operatorname{cag} A 3^{\prime}$ 'variable region.
Abl kinases (Selbach et al., 2002; Stein et al., 2002; Poppe et al., 2007; Tammer et al., 2007). H. pylori strains possessing the cag PAI induce pro-inflammatory interleukin (IL)-8 secretion from gastric epithelial cells (Tummuru et al., 1995; Censini et al., 1996; Keates et al., 1997); this is thought to be central to the induction of local gastric neutrophil infiltration and so central to disease pathogenesis (Robinson et al., 2007). Recently, H. pylori cell-wall peptidoglycan, translocated into the cytosol via the type IV secretion system, has been shown to activate $N F-\kappa B$ via Nod1 leading to IL-8 secretion (Viala et al., 2004), and CagA, independently of phosphorylation, has also been shown to be involved in IL-8 induction during prolonged incubation (Brandt et al., 2005; Kim et al., 2006).

Phosphorylation of CagA occurs on tyrosine residues, within EPIYA motifs, present within the variable region 
(VR) of the protein. Increasing the number of EPIYA motifs leads to an increase in the level of CagA phosphorylation (Higashi et al., 2002a; Argent et al., 2004, 2008a; Zhang et al., 2005; Naito et al., 2006). The EPIYA motifs have been classified into four types, A, B, C and D (Higashi et al., 2002a), based on the amino acid sequences following each type and all types can be phosphorylated in vitro. The A and B motifs can be found in all strains regardless of geographical location. The $\mathrm{C}$ motif is additionally present within 'western' strains and the D motif within strains from East Asia (predominantly Korea, China and Japan). The phosphorylated C and D motifs have been shown to interact with SHP-2 phosphatase, leading to cellular rearrangements and the formation of long cellular protrusions in AGS gastric epithelial cells (Higashi et al., 2002b). However, the D motif is more effective at interacting with SHP-2 (Azuma et al., 2004; Higashi et al., 2004; Naito et al., 2006) as it contains the SHP-2 consensus binding motif $\mathrm{pY}(\mathrm{S} / \mathrm{T} / \mathrm{A} / \mathrm{V} / \mathrm{I}) \mathrm{X}(\mathrm{V} / \mathrm{I} /$ $\mathrm{L}) \mathrm{X}(\mathrm{W} / \mathrm{F})$ (De Sousa et al., 2002), whereas the C motif lacks the final part of this motif. The phosphorylated A and B motifs appear to bind to C-terminal Src kinase (Csk), leading to downregulation of Src kinase activity (Tsutsumi et al., 2003; Naito et al., 2006). This may serve to regulate phosphorylation of CagA.

We aimed to determine the effects of $H$. pylori strains with different numbers and types of CagA tyrosine phosphorylation motifs (TPMs), during short and more prolonged incubation times, on IL-8 secretion, and to compare the prevalence of different CagA TPMs between western and East Asian strains.

\section{METHODS}

H. pylori strains. $H$. pylori strains were obtained from pinch biopsies during routine endoscopy from patients attending Queen's Medical Centre, Nottingham, UK, with full consent and under ethical approval. Biopsies were spread across blood-agar plates (Oxoid) and 20 single colonies were isolated per strain. Single-colony isolates of H. pylori strains were genotyped for the cagA VR (Rudi et al., 1998). Isolates that displayed variation in the size of the $\operatorname{cag} A$ VR were shown to be present within the same strain background by randomly amplified polymorphic DNA (RAPD)-PCR (data not shown) as described previously (Akopyanz et al., 1992). Nucleotide sequencing of cagA VRs showed that variation was due to intragenomic recombination of a 102 nt region encoding the $\mathrm{C}$ motif. $H$. pylori strains were also obtained from populations in western Scotland (ElOmar et al., 2000a, b; Argent et al., 2008a) and China (Zhang et al., 2005).

AGS gastric epithelial cell co-culture and IL-8 ELISA. AGS cells were seeded into six-well plates at a density of $3 \times 10^{5}$ cells per well and incubated at $37{ }^{\circ} \mathrm{C}$ in a $5 \% \mathrm{CO}_{2}$ air-humidified atmosphere for 2 days before the addition of $H$. pylori strains (m.o.i. 100) and further incubation for 6-48 h. For comparison of $H$. pylori strains expressing western CagA with those expressing East Asian CagA, three wells per six-well plate were inoculated with a western strain and the other three wells were inoculated with an East Asian strain. After incubation, the medium was removed, centrifuged at $15000 \mathrm{~g}$ for $10 \mathrm{~min}$ and the amount of IL- 8 secreted into the medium was determined using a DuoSet human IL-8 ELISA kit (R\&D Systems; Argent et al., 2004).

TPM patterns in CagA VRs. The sequences of the CagA VR from 980 strains were obtained from GenBank and from publications where no GenBank accession numbers have been provided (Covacci et al., 1993; Tummuru et al., 1993; Evans et al., 1998; Yamaoka et al., 1998; Dong et al., 2002; Stein et al., 2002; Argent et al., 2004, 2005; Zhu et al., 2005; Devi et al., 2006; Choi et al., 2007; Reyes-Leon et al., 2007). The GenBank accession numbers used were: AB003397, AB015404-AB015415, АB017921-AB017923, АB057003-AB057105, AB089318, AB090073-AB090154, AB110957-AB110974, AB116733AB116768, AB120416-AB120426, AB190932-AB190957, AB246731AB246743, AB267217-AB267264, AE000511, AE001439, AF043487AF043490, AF083352, AF202973, AF222807-AF222809, AF247651, AF249275, AF282853, AF289432-AF289464, AF367250, AF367251, AF427098-AF427100, AF479032, AJ832140-AJ832149, AM279288AM279335, AM292553-AM292599, AM295786-AM295791, AY121840, AY330637, AY330639, AY330642, AY330644, AY550124, AY571191, AY769770-AY769772, CP000241, DQ011619, DQ011620, DQ306710, DQ067454, DQ091000, DQ812087, DQ985738, EF199548, EF202844, EF202845, EF450136-EF450167 and EF444936-EF444938. Strains that displayed microevolution in cagA (usually duplication or deletion of TPMs between different isolates of otherwise identical strains) were excluded from the analysis.

Statistics. Analysis of IL-8 secretion data was performed using Student's $t$-test. Analysis of the occurrence of different types of CagA VR was expressed as odds ratios (OR) with a $95 \%$ confidence interval (CI).

\section{RESULTS AND DISCUSSION}

Recently, CagA has been shown to play a role in the induction of IL-8 secretion from gastric epithelial cells during prolonged periods of incubation $(\geqslant 36 \mathrm{~h})$. This is independent of CagA phosphorylation, but dependent upon the region possessing the TPMs (Brandt et al., 2005). However, no data exist to show whether increasing the number of TPMs is accompanied by an increase in IL-8 secretion. The phenomenon of microevolution has been used to describe a genetic change in a single gene in one strain from an individual's stomach that potentially leads to a change in phenotype. This has been demonstrated for cagA from western strains (Aras et al., 2003; Carroll et al., 2004; Kim et al., 2006; Panayotopoulou et al., 2007; ReyesLeon et al., 2007; Argent et al., 2008a; and in GenBank accession nos EF450138, EF450139 and EF450145EF450149). It occurs commonly in these western populations (up to $\sim 20 \%$ of strains) in which there is either intragenomic duplication or deletion of a $102 \mathrm{nt}$ region encoding the $\mathrm{C}$ motif, and we have isolated 14 examples of cagA microevolution resulting in single or double duplication/deletion of $\mathrm{C}$ motifs in $H$. pylori strain collections from the UK (Nottingham and western Scotland; Argent et al., 2008a). We used this phenomenon to investigate whether increased numbers of $\mathrm{C}$ motifs, which increase the extent of CagA phosphorylation (Argent et al., 2004, 2008a), led to increased IL-8 secretion from AGS gastric epithelial cells. We co-cultured seven $H$. pylori paired isolates that displayed microevolution within cagA with 
AGS cells for 6 or $36 \mathrm{~h}$ and found that increasing the number of $\mathrm{C}$ motifs significantly increased the amount of IL-8 secreted from the cells after prolonged incubation in all cases (Fig. 1). Interestingly, for five of these strains, there was also a significant increase in IL-8 secretion after only $6 \mathrm{~h}$ incubation. We have shown previously that the number of EPIYA motifs had no effect on IL-8 secretion after $6 \mathrm{~h}$ co-culture (Argent et al., 2004) although these data were obtained using independent strains from South Africa, rather than microevolved isolates, and we also found that IL- 8 secretion after $6 \mathrm{~h}$ co-culture was unaffected between wild-type strains and their isogenic cagA mutants (Argent et al., 2008b). However, Brandt et al. (2005) showed that isogenic cagA mutants induced significantly less IL-8 secretion than their parental strain after $9 \mathrm{~h}$ co-culture, suggesting that CagA may play a role in IL-8 induction, even during short incubation periods.

East Asian H. pylori strains possess a D motif within CagA in place of the western $\mathrm{C}$ motif and this has been shown to be a potent inducer of SHP-2 phosphatase (Higashi et al., 2002a; Azuma et al., 2004). Co-culture of four western strains from Scotland expressing CagA with $\mathrm{ABC}$ motifs alongside four East Asian strains from China expressing CagA with ABD motifs with AGS cells for 6 or $48 \mathrm{~h}$ revealed that, in each case, East Asian strains induced significantly more IL-8 secretion (Fig. 2). When the experiment was repeated by incubating each Scottish strain with a different Chinese strain to the initial experiment (see Fig. 2), there was a similar result - the strain with an $\mathrm{ABD}$ CagA induced significantly more IL-8 secretion than the strain with an $\mathrm{ABC}$ CagA. In total, we assessed $H$. pylori induction of IL-8 secretion from AGS cells from seven Scottish and six Chinese strains and found that after $6 \mathrm{~h}$ incubation there was no significant difference $(P=0.35)$ between strains with an $\mathrm{ABC}$ motif $(630 \pm 143$ pg IL-8 $\left.\mathrm{ml}^{-1}\right)$ and those with an ABD motif $(721 \pm 201 \mathrm{pg}$ IL-8 $\mathrm{ml}^{-1}$ ), but after $48 \mathrm{~h}$ incubation, a time point known to be important in CagA-induced IL-8 secretion (Brandt et al., $2005)$, there was a significant $(P=0.015)$ increase in IL-8 secretion for the strains with an ABD motif $(2512 \pm 1166$ vs $4617 \pm 1389 \mathrm{pg} \mathrm{IL}-8 \mathrm{ml}^{-1}$ ). Although these experiments were conducted using separate strains, rather than using isogenic mutants in which the $\mathrm{C}$ and $\mathrm{D}$ motifs have been switched, the data suggest that the D motif, as well as being more effective at activating SHP-2, is also capable of inducing more IL-8 secretion than the C motif. CagA has been shown to cause sustained activation of Erk following activation of SHP-2 (Higashi et al., 2004) and CagAinduced activation of IL- 8 occurs via the Erk pathway but appears to be independent of SHP-2 (Brandt et al., 2005). It is unclear, therefore, how the D motif may lead to greater IL-8 secretion, but this may be due to the differences in sequence surrounding the EPIYA motifs and in the regions between these motifs. The increase in IL- 8 secretion induced by the $\mathrm{D}$ motif has important implications in vivo, as it suggests that East Asian $H$. pylori strains may induce more inflammation within the stomach and this may lead to an increase in atrophic gastritis and gastric cancer. The incidence of these diseases is significantly higher in Japanese patients infected with East Asian strains compared with Japanese patients infected with western strains (Azuma et al., 2004).

A comparison of the western and East Asian CagA VR types from 980 strains (Table 1 ) revealed that there were 22 different western types and 14 different East Asian types, with $\mathrm{ABC}(63.3 \%)$ and $\mathrm{ABD}(88.3 \%)$ being the most common in each group, respectively. It has previously been observed that the ABD type of CagA is most common in East Asia, whereas western forms of CagA display more variation (Hatakeyama, 2006), but this has not previously

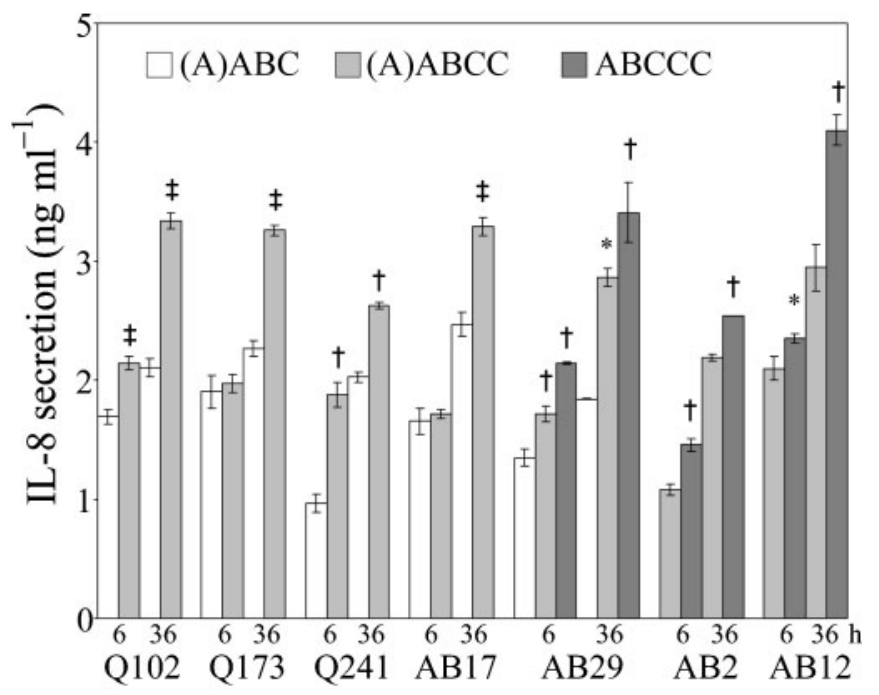

Fig. 1. Increasing the number of CagA $C$ motifs in isolates of microevolved western strains induces significantly more IL-8 secretion. AGS cells were co-cultured with isolates of $H$. pylori strains from Nottingham (Q102, Q173 and Q241) and from Scotland (AB17, $A B 29, A B 2$ and $A B 12)$ that displayed variations in the numbers of CagA $C$ motifs but were otherwise identical by RAPD-PCR (data not shown), in triplicate for 6 and $36 \mathrm{~h}$, before the amount of IL- 8 secreted into the medium was determined by ELISA. Open bars, CagA $A B C$ (strain Q173, AABC); light-grey bars, CagA ABCC (strain Q173, AABCC); darkgrey bars, CagA ABCCC. *, $P<0.05$; $\dagger, P$ $<0.01 ; \ddagger, P<0.001$ (Student's $t$-test). The experiment was performed in triplicate with similar findings in each case. Results are shown as means \pm SD. 


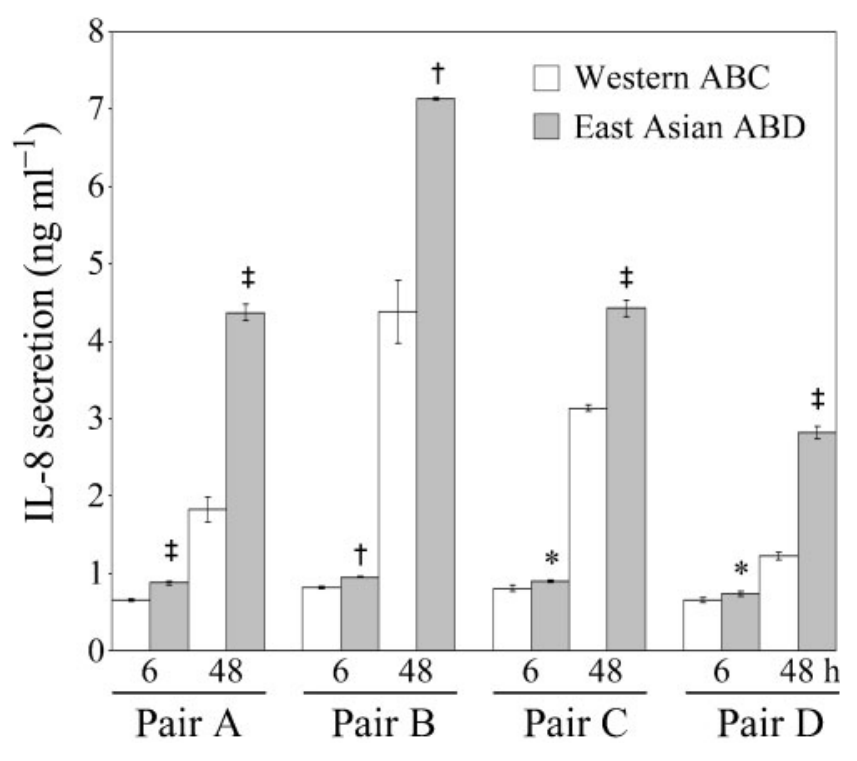

Fig. 2. East Asian $H$. pylori strains induce significantly more IL-8 secretion than western strains. AGS cells were co-cultured with $H$. pylori strains from Scotland (ABC motif; open bars) alongside $H$. pylori strains from China (ABD motif; shaded bars) in triplicate on four six-well plates (four randomly chosen pairs: pair A, Scottish strain $A B 1$ and Chinese strain Z7; pair $B$, Scottish strain $A B 3$ and Chinese strain Z19; pair C, Scottish strain AB6 and Chinese strain Z23; pair D, Scottish strain AB11 and Chinese strain Z28) for 6 and $48 \mathrm{~h}$ before the amount of IL-8 secreted into the medium was determined by ELISA. In all cases, the East Asian ABD strain induced significantly more IL-8 secretion than the western $A B C$ strain after $48 \mathrm{~h}$ incubation $(P<0.01)$ and even after $6 \mathrm{~h}$ incubation $(P<0.05)$. ${ }^{*}, P<0.05 ; \dagger, P<0.01 ; \ddagger, P<0.001$ (Student's $t$-test). When the experiment was repeated by changing the pairs (first pair: strains $A B 1$ and $Z 19$; second pair: strains $A B 3$ and Z23; third pair: strains $A B 6$ and Z28; fourth pair: strains $A B 11$ and Z7), the Chinese strain again induced significantly more IL-8 secretion than the Scottish strain. Each experiment was performed in duplicate with similar findings. Results are shown as means \pm SD.

been quantified. Analysis of those strains possessing CagA with two or more $\mathrm{C}$ or D motifs revealed that western strains were significantly more likely to have additional C motifs than East Asian strains were to have additional D motifs $(\mathrm{OR}=50.2,95 \%$ CI 18.3-137.5). There were only four $(0.7 \%)$ East Asian strains with two D motifs (ABDD, ABDBD and ABDABD types) compared with 109 (26.3\%) western strains with two or more C motifs (Table 1). Analysis of those strains possessing CagA with three or more A or B motifs showed that East Asian strains were significantly more likely to have additional copies of these motifs than western strains ( $\mathrm{OR}=2.53,95 \% \mathrm{CI} 1.37-4.66)$.

These data clearly show that strains from East Asia predominantly possess the $\mathrm{ABD}$ motif: they rarely have additional $\mathrm{D}$ motifs and are more likely to gain additional A or B motifs. The D motif has greater affinity to bind SHP-2, cause cytoskeletal alterations (Higashi et al., 2002a;
Table 1. Prevalence of different types of CagA TPM

\begin{tabular}{|c|c|c|c|}
\hline \multicolumn{2}{|c|}{ Western CagA TPMs } & \multicolumn{2}{|c|}{ East Asian CagA TPMs } \\
\hline Type & Number & Type & Number \\
\hline $\mathrm{AB}^{*}$ & 19 & $\mathrm{AABD}$ & 4 \\
\hline $\mathrm{AAB}^{\star}$ & 1 & AAABD & 3 \\
\hline $\mathrm{AABC}$ & 3 & $\mathrm{AAAD}$ & 1 \\
\hline AABCC & 1 & $\mathrm{ABBD}$ & 28 \\
\hline AAABC & 1 & $\mathrm{ABD}$ & 500 \\
\hline AAC & 1 & ABDD & 2 \\
\hline $\mathrm{ABB}^{*}$ & 1 & ABDABD & 1 \\
\hline $\mathrm{ABBC}$ & 3 & ABDBD & 1 \\
\hline $\mathrm{ABC}$ & 262 & ABABD & 7 \\
\hline $\mathrm{ABCC}$ & 81 & $\mathrm{AeBD} \dagger$ & 5 \\
\hline $\mathrm{ABCCC}$ & 19 & $\mathrm{AD}$ & 8 \\
\hline ABCCCC & 1 & $\mathrm{BD}$ & 4 \\
\hline ABCCCCC & 2 & $\mathrm{BBD}$ & 1 \\
\hline $\mathrm{ABCABC}$ & 1 & BABD & 1 \\
\hline $\mathrm{ABCB}$ & 1 & & \\
\hline $\mathrm{ABCBCC}$ & 1 & & \\
\hline $\mathrm{ABABC}$ & 1 & & \\
\hline $\mathrm{AeBC} \dagger$ & 5 & & \\
\hline $\mathrm{AC}$ & 4 & & \\
\hline ACC & 2 & & \\
\hline ACCC & 1 & & \\
\hline $\mathrm{BC}$ & 3 & & \\
\hline
\end{tabular}

${ }^{\star}$ These types of CagA lack a C or D motif but the sequence following the $\mathrm{B}$ motif indicates that these are western CagA, even though some of the strains possessing these motifs were isolated from East Asian patients.

$\dagger$ These types of CagA possess a tandem EPIYA-B motif: E(S/ $\mathrm{P}) \operatorname{IYAEPIY}(\mathrm{A} / \mathrm{T}) \mathrm{QVAKKV}(\mathrm{N} / \mathrm{S}) \mathrm{A}$. One of the five western strains possessed an unassignable motif (EPIYAKVDDL) before the B motif.

Azuma et al., 2004), and may induce greater levels of IL-8 than the corresponding $\mathrm{C}$ motif (Fig. 2). It is tempting, therefore, to speculate that there is no requirement for East Asian CagA to increase the number of D motifs, whereas there may be more pressure for western CagA to increase the number of $\mathrm{C}$ motifs due to weaker affinity for SHP-2 binding. Increasing the number of $\mathrm{C}$ motifs increases the amount of SHP-2 binding to CagA, although an ABCCC type of CagA appears to bind less SHP-2 than an ABD type (Higashi et al., 2002a). Whereas the $\mathrm{C}$ and $\mathrm{D}$ motifs can bind SHP-2, the A and B motifs have been reported to bind and activate Csk, which then downregulates Src kinase activity to regulate the phosphorylation of CagA (Tsutsumi et al., 2003; Naito et al., 2006). The increase in A and B motifs found in East Asian CagA may serve to regulate the activity of the more active D motif (50\% of East Asian strains with two D motifs have additional A and B motifs, compared with only $2.7 \%$ of western strains; $\mathrm{OR}=35.3$, $95 \% \mathrm{CI}=3.6-342.4$ ). However, increasing the number of $\mathrm{A}$ or B motifs in East Asian CagA had little effect on the binding of Csk, except in two strains that possessed two BD motifs, where there was a significant increase in Csk 
binding activity (Naito et al., 2006). It is significant that an $\mathrm{ABD}$ motif is present in $88.3 \%$ of East Asian strains $(\mathrm{OR}=4.4,95 \% \mathrm{CI} 3.2-6.1)$, supporting the notion that this is the optimal motif for CagA in East Asian strains.

In summary, East Asian CagA predominantly possesses the ABD type and duplication of the D motif is rare, whilst duplication of the A and/or B motif is more common than in western CagA, which frequently duplicates its $\mathrm{C}$ motif. This suggests that the highly active $\mathrm{ABD}$ form does not need to increase its virulence, whereas the $\mathrm{ABC}$ motif, which has much less affinity for SHP-2 and, as we have shown here, is a less potent inducer of IL-8, has a requirement, possibly under certain selective pressures within the stomach, to increase the number of $\mathrm{C}$ motifs. The dynamic capacity of western CagA, which may also permit it to decrease the number of $\mathrm{C}$ motifs, could allow $H$. pylori to regulate its virulence and thereby induce lower level pro-inflammatory and pro-oncogenic signalling.

\section{ACKNOWLEDGEMENTS}

This work was funded in part by Cancer Research UK, by the digestive disorders foundation CORE and by the School of Medical and Surgical Sciences, Queen's Medical Centre, University of Nottingham, UK. J.L.H. was funded by a studentship from the Medical Research Council, UK.

\section{REFERENCES}

Akopyanz, N., Bukanov, N. O., Westblom, T. U., Kresovich, S. \& Berg, D. E. (1992). DNA diversity among clinical isolates of Helicobacter pylori detected by DNA-based RAPD fingerprinting. Nucleic Acids Res 20, 5137-5142.

Aras, R. A., Lee, Y., Kim, S.-K., Israel, D., Peek, R. M., Jr \& Blaser, M. J. (2003). Natural variation in populations of persistently colonizing bacteria affect human host cell phenotype. J Infect Dis 188, 486-496.

Argent, R. H., Kidd, M., Owen, R. J., Thomas, R. J., Limb, M. C. \& Atherton, J. C. (2004). Determinants and consequences of different levels of CagA phosphorylation for clinical isolates of Helicobacter pylori. Gastroenterology 127, 514-523.

Argent, R. H., Zhang, Y. \& Atherton, J. C. (2005). Simple method for determination of the number of Helicobacter pylori CagA variableregion EPIYA tyrosine phosphorylation motifs by PCR. J Clin Microbiol 43, 791-795.

Argent, R. H., Thomas, R. J., Aviles-Jimenez, J., Letley, D. P., Limb, M. C., EI-Omar, E. M. \& Atherton, J. C. (2008a). Toxigenic Helicobacter pylori infection precedes gastric hypochlorhydria in cancer relatives, and H. pylori virulence evolves in these families. Clin Cancer Res 14, 2227-2235.

Argent, R. H., Thomas, R. J., Letley, D. P., Rittig, M. G., Hardie, K. R. \& Atherton, J. C. (2008b). Functional association between the Helicobacter pylori virulence factors VacA and CagA. J Med Microbiol 57, 145-150.

Atherton, J. C. (2006). The pathogenesis of Helicobacter pylori-induced gastro-duodenal diseases. Annu Rev Pathol Mech Dis 1, 63-96.

Azuma, T., Yamazaki, S., Yamakawa, A., Ohtani, M., Muramatsu, A., Suto, H., Ito, Y., Dojo, M., Yamazaki, Y. \& other authors (2004). Association between diversity in the Src homology 2 domaincontaining tyrosine phosphatase binding site of Helicobacter pylori
CagA protein and gastric atrophy and cancer. J Infect Dis 189, 820-827.

Blaser, M. J., Perez-Perez, G. I., Kleanthous, H., Cover, T. L., Peek, R. M., Chyou, P. H., Stemmermann, G. N. \& Nomura, A. (1995). Infection with Helicobacter pylori strains possessing cagA is associated with an increased risk of developing adenocarcinoma of the stomach. Cancer Res 55, 2111-2115.

Brandt, S., Kwok, T., Hartig, R., König, W. \& Backert, S. (2005). NF$\kappa \mathrm{B}$ activation and potentiation of proinflammatory responses by the Helicobacter pylori CagA protein. Proc Natl Acad Sci U S A 102, 93009305.

Carroll, I. M., Ahmed, N., Beesley, S. M., Khan, A. A., Ghousunnissa, S., Moráin, C. A. O., Habibullah, C. M. \& Smyth, C. J. (2004). Microevolution between paired antrum and corpus Helicobacter pylori isolates recovered from individual patients. J Med Microbiol 53, 669-677.

Censini, S., Lange, C., Xiang, Z., Crabtree, J. E., Ghiara, P., Borodovsky, M., Rappuoli, R. \& Covacci, A. (1996). cag, a pathogenicity island of Helicobacter pylori, encodes type I-specific and disease-associated virulence factors. Proc Natl Acad Sci U S A 93, 14648-14653.

Choi, K. D., Kim, N., Lee, D. H., Kim, J. M., Kim, J. S., Jung, H. C. \& Song, I. S. (2007). Analysis of the $3^{\prime}$ variable region of the cagA gene of Helicobacter pylori isolated in Koreans. Dig Dis Sci 52, 960-966.

Covacci, A., Censini, S., Bugnoli, M., Petracca, R., Burroni, D., Macchia, G., Massone, A., Papini, E., Xiang, Z. \& other authors (1993). Molecular characterization of the $128-\mathrm{kDa}$ immunodominant antigen of Helicobacter pylori associated with cytotoxicity and duodenal ulceration. Proc Natl Acad Sci U S A 90, 5791-5795.

De Sousa, D., Fabri, L. J., Nash, A., Hilton, D. J., Nicola, N. A. \& Baca, M. (2002). SH2 domains from suppressor of cytokine signaling-3 and protein tyrosine phosphatase SHP-2 have similar binding specificities. Biochemistry 41, 9229-9236.

Devi, S. M., Ahmed, I., Khan, A. A., Rahman, S. A., Alvi, A., Sechi, L. A. \& Ahmed, N. (2006). Genomes of Helicobacter pylori from native Peruvians suggest admixture of ancestral and modern lineages and reveal a western type cag-pathogenicity island. BMC Genomics 7, 191.

Dong, Q., O'Sullivan, M., Hall, W., Herra, C., Kean, C., O'Morain, C. \& Buckley, M. (2002). Identification of a new segment involved in cagA $3^{\prime}$ region variation of Helicobacter pylori. FEMS Immunol Med Microbiol 33, 51-55.

El-Omar, E. M., Carrington, M., Chow, W.-H., McColl, K. E. L., Bream, J. H., Young, H. A., Herrera, J., Lissowska, J., Yuan, C.-C. \& other authors (2000a). Interleukin-1 polymorphisms associated with increased risk of gastric cancer. Nature 404, 398-402.

El-Omar, E. M., Oien, K., Murray, L. S., El-Nujumi, A., Wirz, A., Gillen, D., Williams, C., Fullarton, G. \& McColl, K. E. L. (2000b). Increased prevalence of precancerous changes in relatives of gastric cancer patients: critical role of $H$. pylori. Gastroenterology 118, 22-30.

Evans, D. J., Jr, Quieroz, D. M. M., Mendes, E. N. \& Evans, D. G. (1998). Diversity in the variable region of Helicobacter pylori cagA gene involves more than simple repetition of a 102-nucleotide sequence. Biochem Biophys Res Commun 245, 780-784.

Hatakeyama, M. (2006). Helicobacter pylori CagA - a bacterial intruder conspiring gastric carcinogenesis. Int J Cancer 119, 12171223.

Higashi, H., Tsutsumi, R., Fujita, A., Yamazaki, S., Asaka, M., Azuma, T. \& Hatakeyama, M. (2002a). Biological activity of the Helicobacter pylori virulence factor $\mathrm{CagA}$ is determined by variation in the tyrosine phosphorylation sites. Proc Natl Acad Sci U S A 99, 14428-14433.

Higashi, H., Tsutsumi, R., Muto, S., Sugiyama, T., Azuma, T., Asaka, M. \& Hatakeyama, M. (2002b). SHP-2 tyrosine phosphatase as an 
intracellular target of Helicobacter pylori CagA protein. Protein Sci 295, 683-686.

Higashi, H., Nakaya, A., Tsutsumi, R., Yokoyama, K., Fujii, Y., Ishikawa, S., Higuchi, M., Takahashi, A., Kurashima, Y. \& other authors (2004). Helicobacter pylori CagA induces Ras-independent morphogenetic response through SHP-2 recruitment and activation. $J$ Biol Chem 279, 17205-17216.

Keates, S., Hitti, Y. S., Upton, M. \& Kelly, C. P. (1997). Helicobacter pylori infection activates NF- $\kappa \mathrm{B}$ in gastric epithelial cells. Gastroenterology 113, 1099-1109.

Kim, S.-Y., Lee, Y.-C., Kim, H. K. \& Blaser, M. J. (2006). Helicobacter pylori CagA transfection of gastric epithelial cells induces interleukin8. Cell Microbiol 8, 97-106.

Naito, M., Yamazaki, T., Tsutsumi, R., Higashi, H., Onoe, K., Yamazaki, S., Azuma, T. \& Hatakeyama, M. (2006). Influence of EPIYA-repeat polymorphism on the phosphorylation-dependent biological activity of Helicobacter pylori CagA. Gastroenterology 130, 1181-1190.

Panayotopoulou, E. G., Sgouras, D. N., Papadakos, K., Kalliaropoulos, A., Papatheodoridis, G., Mentis, A. F. \& Archimandritis, A. J. (2007). Strategy to characterize the number and type of repeating EPIYA phosphorylation motifs in the carboxy terminus of CagA protein in Helicobacter pylori clinical isolates. J Clin Microbiol 45, 488-495.

Poppe, M., Feller, S. M., Römer, G. \& Wessler, S. (2007). Phosphorylation of Helicobacter pylori CagA by c-Abl leads to cell motility. Oncogene 26, 3462-3472.

Reyes-Leon, A., Atherton, J. C., Argent, R. H., Puente, J. L. \& Torres, J. (2007). Heterogeneity in the activity of Mexican Helicobacter pylori strains in gastric epithelial cells and its association with diversity in the cagA gene. Infect Immun 75, 3445-3454.

Robinson, K., Argent, R. H. \& Atherton, J. C. (2007). The inflammatory and immune response to Helicobacter pylori infection. Best Pract Res Clin Gastroenterol 21, 237-259.

Rudi, J., Kolb, C., Maiwald, M., Zuna, I., von Herbay, A., Galle, P. R. \& Stremmel, W. (1998). Diversity of Helicobacter pylori vacA and cagA genes and relationship to VacA and CagA protein expression, cytotoxin production, and associated diseases. J Clin Microbiol 36 944-948.
Selbach, M., Moese, S., Hauck, C. R., Meyer, T. F. \& Backert, S. (2002). Src is the kinase of the Helicobacter pylori CagA protein in vitro and in vivo. J Biol Chem 277, 6775-6778.

Stein, M., Bagnoli, F., Halenbeck, R., Rappuoli, R., Fantl, W. J. \& Covacci, A. (2002). c-Src/Lyn kinases activate Helicobacter pylori CagA through tyrosine phosphorylation of the EPIYA motifs. Mol Microbiol 43, 971-980.

Tammer, I., Brandt, S., Hartig, R., König, W. \& Backert, S. (2007). Activation of Abl by Helicobacter pylori: a novel kinase for CagA and crucial mediator of host cell scattering. Gastroenterology 132, 1309-1319.

Tsutsumi, R., Higashi, H., Higuchi, M., Okada, M. \& Hatakeyama, M. (2003). Attenuation of Helicobacter pylori CagA-SHP-2 signaling by interaction between CagA and C-terminal Src kinase. J Biol Chem 278, 3664-3670.

Tummuru, M. K., Cover, T. L. \& Blaser, M. J. (1993). Cloning and expression of a high-molecular-mass major antigen of Helicobacter pylori: evidence of linkage to cytotoxin production. Infect Immun 61, 1799-1809.

Tummuru, M. K., Sharma, S. A. \& Blaser, M. J. (1995). Helicobacter pylori picB, a homologue of the Bordetella pertussis toxin secretion protein, is required for induction of IL-8 in gastric epithelial cells. Mol Microbiol 18, 867-876.

Viala, J., Chaput, C., Boneca, I. G., Cardona, A., Girardin, S. E., Moran, A. P., Athman, R., Mémet, S., Huerre, M. R. \& other authors (2004). Nod1 responds to peptidoglycan delivered by the Helicobacter pylori cag pathogenicity island. Nat Immunol 5, 1166-1174.

Yamaoka, Y., Kodama, T., Kashima, K., Graham, D. Y. \& Sepulveda, A. R. (1998). Variants of the $3^{\prime}$ region of the cagA gene in Helicobacter pylori isolates from patients with different $H$. pylori-associated diseases. J Clin Microbiol 36, 2258-2263.

Zhang, Y., Argent, R. H., Letley, D. P., Thomas, R. J. \& Atherton, J. C. (2005). Tyrosine phosphorylation of CagA from Chinese Helicobacter pylori isolates in AGS gastric epithelial cells. J Clin Microbiol 43, 786790.

Zhu, Y.-L., Zheng, S., Du, Q., Qian, K.-D. \& Fang, P.-C. (2005). Characterization of CagA variable region of Helicobacter pylori isolates from Chinese patients. World J Gastroenterol 11, 880-884. 